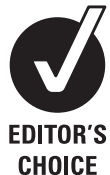

CHOICE
- Additional appendices are published online only. To view these files, please visit the journal online (http://heart.bmj. com)

${ }^{1}$ Department of Cardiac Electrophysiology, The Heart Hospital, UCLH, London, UK ${ }^{2}$ Department of Radiology, The Heart Hospital, UCLH, London, UK

${ }^{3}$ Department of Medical Physics and Bioengineering, The Heart Hospital, UCLH, London, UK

Correspondence to Dr Anthony Chow, Department of Cardiac Electrophysiology, The Heart Hospital, UCLH Trust, 16-18 Westmoreland Street, London W1G 8PH, UK: anthony.chow@uclh.nhs.uk

Accepted 9 September 2010 Published Online First 29 October 2010

\title{
Improving safety in the electrophysiology laboratory using a simple radiation dose reduction strategy: a study of 1007 radiofrequency ablation procedures
}

\author{
Dominic P S Rogers, ${ }^{1}$ Fiona England, ${ }^{2}$ Konstantin Lozhkin, ${ }^{3}$ Martin D Lowe, \\ Pier D Lambiase, ${ }^{1}$ Anthony W C Chow ${ }^{1}$
}

\begin{abstract}
Background The use of fluoroscopic screening involves exposure to ionising radiation for both patients and operators.

Objective To assess the effects of radiation dose reduction manoeuvres (DRM) during radiofrequency ablation (RFA) procedures.

Design Prospective study of DRM.

Setting Tertiary cardiac centre.
\end{abstract}

Interventions Two DRM were combined: removal of the secondary radiation grid and programming an ultra-low pulsed fluoroscopy rate. These methods were assessed using an anthropomorphic phantom model to measure skin entrance dose rates. Procedures were classified as complex (ablation of atrial fibrillation, ventricular tachycardia or complex congenital heart disease arrhythmias) or simple (all other RFA)

Main outcome measures Dose area product and screening times were compared for ablations performed before and after DRM. Equivalent doses to organs and malignancy risk were determined by computer modelling. Results Over a 39-month period, 1007 ablation procedures were performed (631 simple, 376 complex) Radiation dose was significantly reduced after DRM for both simple $\left(20.4 \pm 26.9 \mathrm{Gycm}^{2}\right.$ vs $8.0 \pm 10.3 \mathrm{Gycm}^{2}$, $\mathrm{p}<0.00001)$ and complex ablations $\left(63.3 \pm 50.1 \mathrm{Gycm}^{2}\right.$ vs $32.8 \pm 31.7 \mathrm{Gycm}^{2}, p<0.00001$ ) with no difference in screening times. The mean lifetime risk of fatal cancer attributable to radiation exposure per million procedures was reduced from 182 to 68 for simple ablations and from 440 to 155 for complex ablations.

Conclusions Significant reductions in radiation exposure during RFA were achieved using simple DRM corresponding to a two-thirds reduction of the risk of excess fatal malignancy.

\section{INTRODUCTION}

The high success rate and low complication risks of radiofrequency ablation (RFA) have led to its increasing use as first line treatment for a number of cardiac arrhythmias. ${ }^{1}$ In recent years, with greater understanding of arrhythmia substrate and development of advanced electroanatomical mapping systems, RFA has emerged as a potential treatment option for more complex arrhythmias including atrial fibrillation $(\mathrm{AF})^{2}$ and ventricular tachycardia. ${ }^{3}$ These factors have contributed to a rapid growth in the numbers of RFA performed worldwide. The majority of RFA procedures use fluoroscopic screening to guide catheter placement and therefore carry risks associated with exposure to ionising radiation including skin injury, ${ }^{4}$ radiationinduced malignancy ${ }^{6-8}$ and genetic effects. ${ }^{7-9}$ These are of particular concern for young patients and for patients undergoing long complex procedures, where high radiation doses have been reported ${ }^{6}$ and in whom repeated procedures may be indicated. ${ }^{10}$ Operators, particularly those performing high volumes of procedures, are also exposed to risks from radiation including malignancy. ${ }^{11}$ Many cardiac catheter laboratories use a secondary radiation grid to improve image resolution by reducing scatter at the image intensifier. Previous authors have shown that the presence of this grid typically doubles the radiation dose. ${ }^{12}$ Since high-definition imaging is not essential for most RFA cases, given the high radioopacity of typical ablation catheters, we hypothesised that removal of the secondary radiation grids could reduce radiation exposure without affecting screening time. In addition, reduction of the fluoroscopy pulse rate could further reduce radiation dose for RFA.

The aims of this study were to assess the radiation reduction resulting from these dose reduction manoeuvres (DRM) using an anthropomorphic phantom model, and to measure total radiation dose for all types of RFA procedures performed routinely in an electrophysiology laboratory before and after DRM.

\section{METHODS}

Radiation doses were assessed in the electrophysiology laboratory before and after DRM. x-Ray imaging was performed using a Philips Integris BH5000 biplane C-arm fluoroscopic unit (Philips Medical Systems, Eindhoven, the Netherlands), equipped with last image hold. The primary tube was positioned under the couch with the image intensifier above and was used for postero-anterior (PA) and right anterior oblique imaging. The lateral tube was used for left anterior oblique projections. Beam filtration consisted of $3.4 \mathrm{~mm} \mathrm{Al}$ to which $0.2 \mathrm{~mm} \mathrm{Cu}$ was added. During fluoroscopy, kilovoltage (40-110 kVp) and tube current (25 mA max) were selected by automatic brightness control. The focus to image intensifier distance was $100 \mathrm{~cm}$. The Philips unit has real-time monitoring of fluoroscopy time and dose area product (DAP) measurement. DAP, the product of total radiation dose and the radiation field area, provides a measure of radiation exposure. DAP metre readings were calibrated using a Keithley 96035 parallel-plate diagnostic ion chamber and a Keithley 35050A Dosimeter (Keithley Instruments, USA) to measure radiation 
dose in the centre of the field while imaging an M1 Leeds test object with a wire matrix (Leeds Test Objects, UK) to measure the area of the field. DAP calibration measurements were done at $105 \mathrm{kVp}, 12 \mathrm{~mA}$ for the AP tube and at $86 \mathrm{kVp}$, $21 \mathrm{~mA}$ for the lateral tube. The Keithley dosimeter and ion chamber were calibrated (with relative uncertainty $2.8 \%$ of the calibration factor) at the John Perry Radiation Metrology Laboratory (London, UK) using a secondary standard system with a current calibration from the Physikalisch-Technische Bundesanstalt (holder of the German national standard for x-ray dosimetry).

\section{Radiation reduction methods}

At baseline (pre-dose reduction), screening fluoroscopy was routinely performed at the factory 'low' setting of 12.5 pulses per second (pps) with a pulse length of $6 \mathrm{~ms}$, and with the secondary radiation grid in situ. In order to reduce radiation dose, we programmed a customised 'ultra-low' fluoroscopy setting at $6.25 \mathrm{pps}$ and removed the grid.

\section{Direct measurement of skin entrance dose rate}

An adult torso anthropomorphic phantom consisting of a natural human skeleton embedded in tissue equivalent rubber (Temex) was used to assess entrance skin dose rate using the Keithley dosimeter and ion chamber, positioned directly underneath the phantom. The position of the ionisation chamber in relation to the x-ray field was verified using fluoroscopy. Dose rates were obtained with the frontal tube in PA, right anterior oblique 30 degrees and, for the lateral tube, left anterior oblique 45 degrees, in keeping with the use of the two tubes in clinical practice. Dose rates were measured under automatic brightness control of $\mathrm{kVp}$ and $\mathrm{mA}$ for the $23 \mathrm{~cm}$ field of view during continuous irradiation for at least $30 \mathrm{~s}$. The measurements were repeated for low and ultra-low fluoroscopy settings both with and without the secondary radiation grid.

\section{Radiation doses in clinical cases}

Following the phantom measurements, DRM were adopted in the electrophysiology laboratory for all RFA procedures. DAP and screening times were examined for the 17 months before and 22 months after DRM. Procedures were classified as 'simple' (ablation of accessory pathway, atrioventricular nodal re-entry tachycardia, typical caval-isthmus dependent atrial flutter, atrioventricular nodal ablation) or 'complex' (ablation of AF, atypical atrial flutter/tachycardias, ventricular tachycardia, or in patients with complex congenital heart disease). Diagnostic electrophysiological procedures were excluded. Conventional multipolar contact catheters were used for mapping and ablation, and all procedures were performed by experienced clinical operators. Non-fluoroscopic three-dimensional mapping systems (CARTO, Biosense Webster, California, USA or Ensite NavX, St Jude Medical, St Paul, Minnesota, USA) were used in conjunction with conventional electrophysiology mapping systems for navigation and electro-anatomical mapping to guide complex ablations.

\section{Calculation of equivalent doses and effective doses}

Equivalent doses to organs for the periods before and after DRM were calculated using the Monte Carlo based PCXMC simulation program (version 1.5), based on skin entrance dose rates and $\mathrm{kVp}$ values determined from the phantom measurements and the mean screening times and proportion of screening spent in each projection measured during the study periods. The PCXMC program was developed by the Radiation and Nuclear Safety
Authority (STUK, Finland) for calculating patients' organ doses in radiography and fluoroscopy. ${ }^{13}$ A large number of individual photon histories are generated to reduce the statistical errors of organ doses, and estimates of the mean values of the energy depositions in the various organs of the phantom are used for calculating equivalent doses for these organs. ${ }^{14}$ Calculations were performed with a focus to skin distance of $80 \mathrm{~cm}$ and the $\mathrm{x}$-ray beam collimated to the heart borders, using a simulation exposure of 10 million photons for each radiographic projection.

The lifetime fatal cancer probability for each organ was calculated as the product of the equivalent dose to the organ and the organ-specific fatal cancer probability coefficient, obtained from the 2007 recommendations of the International Commission on Radiological Protection (ICRP) approved in March $2007 .{ }^{15}$ The effective dose for each radiographic projection was calculated as the sum of the equivalent organ doses determined by the computer model, multiplied by the appropriate tissue weighting factors, ${ }^{15}$ and body effective dose was computed as the sum of effective doses for the three radiographic projections. The excess lifetime risk of malignancy (attributable to the RFA radiation) was then determined using the population-averaged probability coefficient $5.5 \%$ per $\mathrm{Sv} .{ }^{15}$ The risk of radiationinduced hereditary effects was computed using doses to the ovaries (doses to the testes were negligible) multiplied by the risk coefficient for hereditary effects $0.2 \%$ per Sv.

\section{Statistical analysis}

Continuous data are presented as mean \pm SD. Ranges are given where appropriate. Unpaired Student's t tests were used to compare baseline characteristics and data before and after radiation reduction. A value of $p<0.05$ was considered significant.

\section{RESULTS}

\section{Phantom measurements}

Table 1 shows measurements of skin entrance dose rates obtained using the phantom. Reductions in dose rate were seen both with removal of the grid and reduction in fluoroscopy pulse rate. When ultra-low settings were combined with removal of the grid, dose rates were 60-65\% lower than baseline measurements in all projections.

\section{Image quality}

Operators were not informed of the changes made to the radiation during cases unless they commented on the quality of imaging. This occurred in only 9 cases and in no cases was there a request to increase the fluoroscopy frame rate; in only 5 postDRM cases (2 simple, 3 complex) was there a request by the operator to replace the grid. Figure 1 shows examples of images of ablation catheters taken with and without the grid.

Table 1 Entrance skin radiation dose rates measured using an anthropomorphic torso phantom representing an average male subject at varying radiographic projections

\begin{tabular}{llllll}
\hline & \multicolumn{2}{l}{$\begin{array}{l}\text { Fluoroscopy } \\
\text { normal }\end{array}$} & & \multicolumn{2}{l}{$\begin{array}{l}\text { Fluoroscopy } \\
\text { ultra-low }\end{array}$} \\
\cline { 2 - 3 } \cline { 6 - 7 } & Grid & No grid & & Grid & No grid \\
\hline Dose rate (PA) (mGy/min) & 5.07 & 2.57 & & 3.56 & 1.77 \\
Dose rate (LA045) (mGy/min) & 4.21 & 2.52 & & 2.86 & 1.71 \\
Dose rate (RA030) (mGy/min) & 5.59 & 2.86 & & 3.71 & 1.91 \\
\hline
\end{tabular}

Measurements were performed with the fluoroscopy at the factory determined 'low' setting and at the customised 'ultra-low' setting, both with and without the grid in situ. The relative uncertainty of dose rate measurements was $2.8 \%$.

$\mathrm{PA}$, posteroanterior projection; LA045, left anterior oblique $45^{\circ}$ projection; RA030, right anterior oblique $30^{\circ}$ projection. 

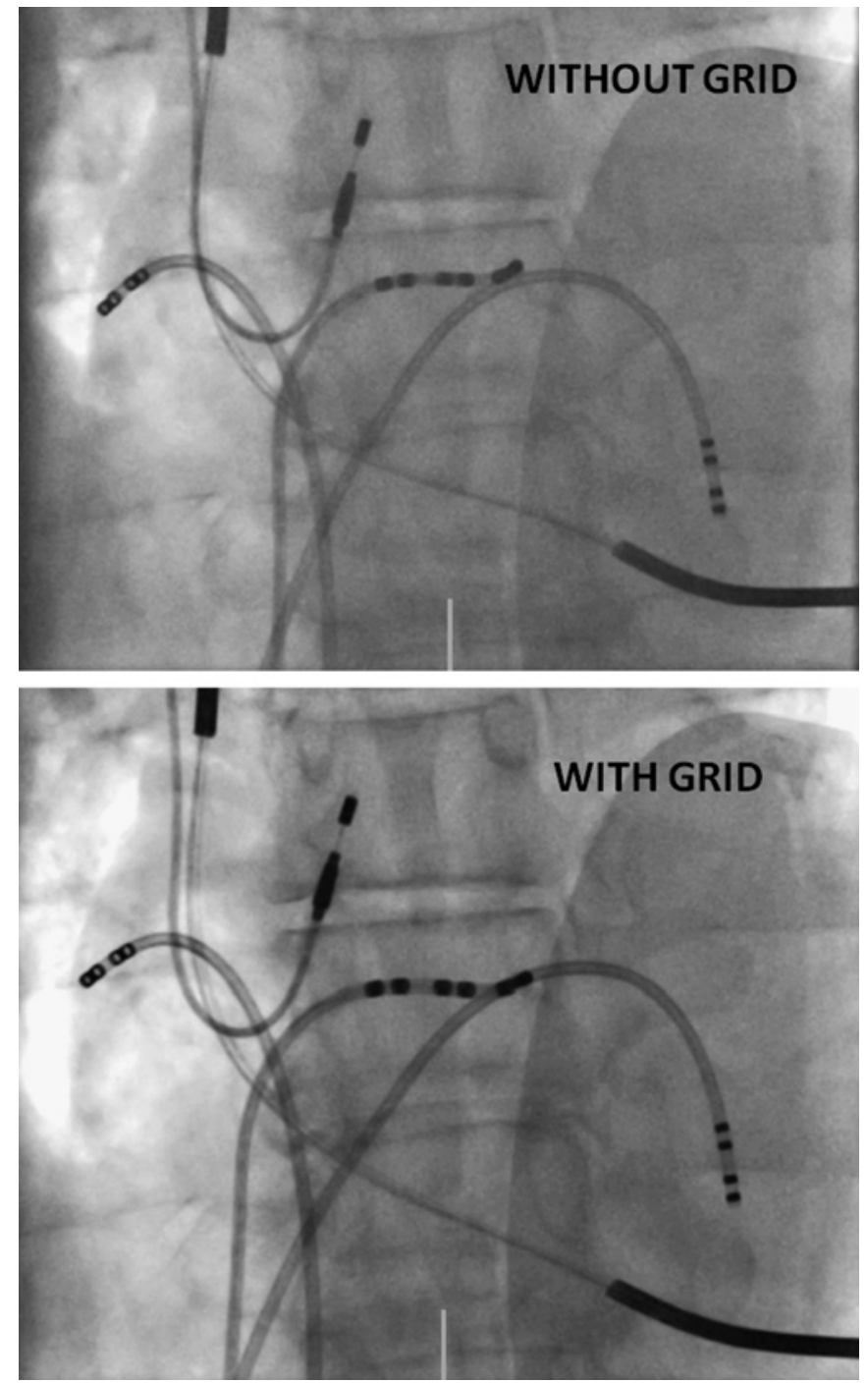

Figure 1 Example images from an ablation case taken to compare image quality without (top) and with (bottom) the secondary radiation grid in situ.

\section{RFA cases}

Between January 2004 and March 2007, 1007 ablation procedures were performed (631 simple, 376 complex). There were no differences in baseline characteristics between the two study periods for both simple and complex procedures (table 2). Standard equipment and catheters were used for simple ablation cases. All complex cases used a three-dimensional mapping system (Carto or NavX) to assist in navigation, mapping and ablation. There were no differences in procedure times for either simple or complex procedures pre- and post-DRM (table 2).

There were significant reductions in radiation DAP after dose reduction for both simple $\left(20.4 \pm 26.9 \mathrm{Gycm}^{2}\right.$ vs $8.0 \pm 10.3$ $\left.\mathrm{Gycm}^{2}, \mathrm{p}<0.00001\right)$ and complex ablations $\left(63.3 \pm 50.1 \mathrm{Gycm}^{2}\right.$ vs $\left.32.8 \pm 31.7 \mathrm{Gycm}^{2}, \mathrm{p}<0.00001\right)$. There was no difference in screening times: $20.3 \pm 16.6 \mathrm{~min}$ vs $21.3 \pm 18.5 \mathrm{~min}(\mathrm{p}=0.55)$ for simple ablations and $55.3 \pm 28.1 \mathrm{~min}$ vs $52.7 \pm 23.3 \mathrm{~min}(\mathrm{p}=0.43)$ for complex ablations (figure 2 ). There was no change in either the success rate or major complication rate for RFA procedures after dose reduction.

\section{Equivalent doses for organs and malignancy risk}

The computed equivalent organ doses for simple and complex ablations are shown in tables AI and AII in the appendix.
Table 2 Baseline characteristics of patients and procedure types

\begin{tabular}{llll}
\hline & Pre-DRM & Post-DRM \\
\hline Simple & & & \\
Subjects (n) & 214 & 417 & \\
Male (\%) & $114(53.3 \%)$ & $219(52.5 \%)$ & \\
Age, years & $53 \pm 17(19-86)$ & $52 \pm 18(17-91)$ & NS \\
Weight, kg & $77 \pm 26(51-127)$ & $79 \pm 18(45-170)$ & NS \\
Procedure time, min & $91.9 \pm 45.5$ & $99.3 \pm 55.3$ & NS
\end{tabular}

Procedure type and number of cases

$\begin{array}{lll}\text { AVNRT/Acc } P & 147 & 257 \\ \text { Flutter } & 53 & 153 \\ \text { AV node ablation } & 14 & 7\end{array}$

\section{Complex}

Subjects (n)

Male (\%)

Age, years

Weight, $\mathrm{kg}$

Procedure time, $\min$

83
$50(60.2 \%)$
$53 \pm 13(25-79)$
$75 \pm 21(62-107)$
$213.4 \pm 71.3$

293

$193(65.9 \%)$

$55 \pm 13(18-85) \quad$ NS

$80 \pm 19(50-158) \quad$ NS

$224.7 \pm 69.3 \quad$ NS

Procedure type and number of cases

$\begin{array}{lll}\text { AF } & 79 & 263 \\ \text { VT } & 3 & 14 \\ \text { cCHD } & 1 & 16\end{array}$

Acc $P$, accessory pathway; $A F$, atrial fibrillation; $A V N R T$, atrioventricular nodal re-entry tachycardia; $\mathrm{CCHD}$, complex congenital heart disease; DRM, dose reduction manoeuvres; VT, ventricular tachycardia.

The organ-specific probability coefficients (ICRP 2007) ${ }^{15}$ are also given along with the calculated fatal malignancy risk for each organ. Estimates of the lifetime risk of excess malignancy derived from the effective dose calculations are shown in table 3.

The risk of fatal malignancy related to x-ray exposure was reduced by $63 \%$ to 68 cases per million procedures for simple RFA and by $65 \%$ to 155 cases per million procedures for complex RFA. Malignancy risk is also given, corrected per hour of screening for comparison with published studies. The estimated risk of hereditary abnormalities post DRM is 6.4 per billion procedures for simple RFA and 14.2 per billion procedures for complex RFA.

\section{DISCUSSION}

The underlying principle of radiological protection, with respect to all medical radiation exposure, is that the radiation dose should be kept as low as reasonably achievable (ALARA). Although image quality provided by fluoroscopic systems has steadily improved over time, the highest resolution and best available image quality may not be required for all types of procedure. This study demonstrates that the use of ultra-low fluoroscopy and removal of the secondary radiation grid can significantly reduce radiation dose in the electrophysiology laboratory without increasing screening times. The effects of these measures on skin entrance dose rate were assessed using a standard phantom model before dose reduction in clinical cases was confirmed by studying a large number of procedures over a period of more than 3 years and comparing DAP measurements. DAP has been shown to have good agreement with other direct measurements of effective radiation dose in diagnostic radiology, ${ }^{16}$ cardiac fluoroscopy ${ }^{17}$ and RFA. ${ }^{7} 18$ Previously reported DAP values for simple RFA range from 11.6 to 251 Gycm $^{2}$ compared to $8.1 \mathrm{Gycm}^{2}$ in this study. ${ }^{18-20}$ Using the phantom data in a computer simulation model to calculate organ dose, we demonstrated an approximate two-thirds reduction in the risk of radiation-induced malignancy, for both simple and complex RFA. 

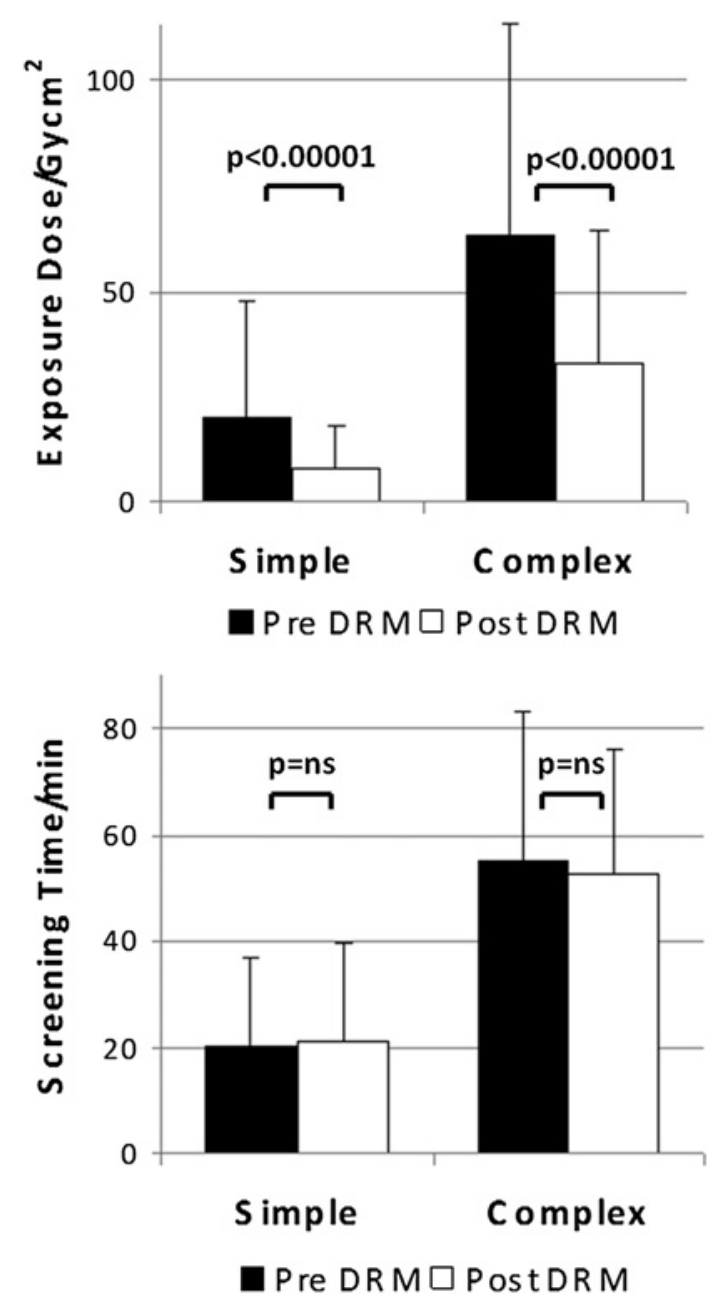

Figure 2 Mean dose area products (top) and screening times (bottom) for ablations performed before and after dose reduction manoeuvres (DRM). Error bars show standard deviations.

\section{Radiation reduction methods}

Alterations to the fluoroscopy pulse rate or pulse width have been used to reduce radiation in cardiac pacing and in electrophysiology studies including ablations. ${ }^{20}$ This reduction in temporal resolution has no effect on image quality and in this study no operator requested that the pulse rate be increased after DRM. Radiation reduction by removal of the grid has been described in various settings including cardiac angiography. ${ }^{12} 2122$ Scatter can be reduced without use of a grid by increasing the air gap to the image intensifier. However, the resultant increased magnification,

Table 3 Mean results for effective dose and the computed lifetime fatal malignancy risk attributable to radiation

\begin{tabular}{llll}
\hline & $\begin{array}{l}\text { Effective } \\
\text { dose }(\mathbf{m S v})\end{array}$ & $\begin{array}{l}\text { Fatal malignancy } \\
\text { risk (typical case) } \\
\left(\times \mathbf{1 0 ^ { 6 } )}\right.\end{array}$ & $\begin{array}{l}\text { Fatal malignancy risk } \\
\text { per hour fluoroscopy } \\
\left(\times 1 \mathbf{1 0}^{6}\right)\end{array}$ \\
\hline Simple & & & \\
Pre-DRM & 3.30 & 182 & 537 \\
Post-DRM & 1.24 & 68 & 185 \\
Complex & & & \\
Pre-DRM & 7.99 & 440 & 506 \\
Post-DRM & 2.83 & 155 & 172
\end{tabular}

The malignancy risk is given both for the average screening time in this study (typical case) and corrected for one hour of fluoroscopy. Data are presented for simple and complex ablations, both before and after dose reduction manoeuvres (DRM). particularly in laboratories with small detector plates as is typical in cardiac laboratories, does not allow visualisation of the entire heart. Physical removal of the grid, which is usually situated in a slot at the front of the image intensifier or attached to its surface, is in our experience a very simple process, taking a few seconds on commonly available systems (eg, manufactured by Philips, Siemens or GE). Similarly, if an operator requests replacement to improve image resolution, this can be achieved within seconds. However, this was only requested in a handful of cases in the present study. Important reductions in radiation dose have been achieved with the development of non-fluoroscopic imaging techniques, particularly in complex ablations, ${ }^{23}$ but this study shows that further reductions can be achieved using simple steps as mapping systems were used throughout this study period.

\section{Comparison of radiation dose and malignancy risk with previous studies}

Published studies show large variations in radiation dose during RFA; many of them describe small numbers of patients where ablation was performed in the setting of a specific radiation study rather than 'real world' cases such as in the present study. A variety of methods have been used to quantify radiation exposure during RFA ${ }^{6-82425}$ and a number of approaches have been adopted to calculate malignancy risks in RFA. ${ }^{6-819}$ Table 4 summarises previously reported RFA studies along with the current study. Even when corrected for screening time, the malignancy risks associated with RFA in this study are up to 15 times lower than in other studies, and represent the lowest of all available published data.

Two studies have specifically investigated radiation doses in AF ablation [table 4]. Lickfett et al recorded doses from 15 procedures, using 50-60 thermoluminescent dosimeters attached to the patients. ${ }^{6}$ Even when the longer screening times in that study $(129.7 \pm 36.7 \mathrm{~min}$ compared to $54.3 \pm 23.5 \mathrm{~min}$ in the present study) are taken into account, the mean effective doses are considerably higher than in the present study. Macle et al used a single electronic dosimeter, which provides an estimate of whole body effective dose based on a measurement at a single point, to record the personal dose equivalent to 43 patients. ${ }^{26}$ The low effective doses reported may have been

Table 4 Mean effective doses and fatal malignancy risk attributable to radiation exposure during simple and complex during radiofrequency ablation

\begin{tabular}{|c|c|c|c|}
\hline & $\begin{array}{l}\text { Mean } \\
\text { effective } \\
\text { dose (mSv) }\end{array}$ & $\begin{array}{l}\text { Fatal malignancy } \\
\text { risk } \times 10^{-6} \\
\text { (typical case) }\end{array}$ & $\begin{array}{l}\text { Fatal malignancy } \\
\text { risk } \times 10^{-6} \\
\text { (per hour } \\
\text { fluoroscopy) }\end{array}$ \\
\hline \multicolumn{4}{|l|}{ Simple ablations } \\
\hline Present study (post-DRM) & 1.24 & 68 & 185 \\
\hline Kovoor et al ${ }^{7}$ & & & $294(F)$ \\
\hline Perisinakis et $a l^{8}$ & 5.67 & & 480 (UK); 650 (USA) \\
\hline Efstathopoulos et al ${ }^{19}$ & 15.2 & 420 & \\
\hline \multirow[t]{2}{*}{ Calkins et $a l^{24}$} & & 730 (M) & 990 (M) \\
\hline & & $720(\mathrm{~F})$ & $980(F)$ \\
\hline \multirow[t]{2}{*}{ Rosenthal et al ${ }^{30}$} & & 2597 (M) & 2642 (M) \\
\hline & & $1407(F)$ & $2010(\mathrm{~F})$ \\
\hline \multicolumn{4}{|l|}{ Complex ablations } \\
\hline Present study (post-DRM) & 2.83 & 155 & 172 \\
\hline Macle et $\left.a\right|^{26}$ & 1.1 & & \\
\hline \multirow[t]{2}{*}{ Lickfett et al ${ }^{6}$} & 27.25 (M) & 2099 (M) & 969 (M) \\
\hline & $18.74(F)$ & 1518 (F) & 700 (F) \\
\hline
\end{tabular}

Previously published studies are presented for comparison. Malignancy risk is also given corrected per hour of fluoroscopic screening to allow for variation in screening times. DRM, dose reduction manoeuvres; $F$, female patients; $M$, male patients. 
underestimated due to the dosimeter, which was positioned over the xiphisternum, not being consistently in the primary beam as its position was not confirmed using screening and AP projection was not exclusively used.

\section{Skin doses and deterministic effect thresholds}

The reference threshold dose to skin for early transient erythema is 2 Gy and the FDA recommends that patient dose be monitored for any procedure which has the potential to exceed $1 \mathrm{~Gy}{ }^{27}$ Previous publications have suggested that this is not uncommon in RFA. In a study of skin doses in 500 RFAs, $5.6 \%$ of patients received enough radiation to reach the 2 Gy threshold dose. ${ }^{5}$ Of the 15 patients undergoing AF ablation in the Lickfett study, all but two patients exceeded the 1 Gy FDA threshold. ${ }^{6}$ In the present study the very low skin entrance dose rates $(1.77 \mathrm{mGy}$ per minute for the PA projection for the average person) mean that for most patients, reaching these thresholds is extremely unlikely.

\section{Clinical implications}

Previous case reports and series have described prolonged screening times and high radiation doses in complex ablation in particular. We have shown that by using simple measures, radiation levels can be kept to levels where the risks of both deterministic and stochastic effects are very small. We report effective doses of $1.24 \mathrm{mSv}$ and $2.83 \mathrm{mSv}$ for simple and complex RFA, respectively. This compares to the average annual background radiation dose in the UK of $2.7 \mathrm{mSv}^{28}$ and a typical dose of approximately $8 \mathrm{mSv}$ associated with an abdominal CT. Another important clinical consideration is radiation exposure for operators and laboratory personnel, who may be involved in a large number of procedures. Although we did not measure operator exposure, reductions in patient dose have been shown to correlate with operator dose, ${ }^{2529}$ and as the majority of operator exposure results from interaction of the x-ray beam with the patient, it is reasonable to expect that reductions in patient entrance dose translate to reduced operator dose. The described DRM could easily be adopted by many other centres, resulting in improved safety for patients and staff in the electrophysiology laboratory.

\section{Limitations}

First, it should be noted that the use of 'effective dose' and its relationship with risk from radiation exposure was developed in population studies (ICRP) and is not recommended for estimating risks in individual patients. Second, although removal and replacement of the grid is extremely simple in the fluoroscopy equipment described in this paper and in other systems with which the authors are familiar, the process differs among systems and may be much more difficult in other laboratories. A further point when considering adopting these DRM in other laboratories is that programming an 'ultra-low' fluoroscopy setting may require the initial help of the medical physics department; however once it has been set up it is straight forward for the radiographer to select this setting for future cases. Finally, an important limitation of this study is that the calculations of effective dose are based on entrance doses measured in a phantom based on an average-sized person. Although this is in keeping with other published studies, it is important to recognise that heavier patients may be exposed to much higher doses.

Competing interests None.

Provenance and peer review Not commissioned; externally peer reviewed.

\section{REFERENCES}

1. Calkins H, Yong P, Miller JM, et al. Catheter ablation of accessory pathways, atrioventricular nodal reentrant tachycardia, and the atrioventricular junction: final results of a prospective, multicenter clinical trial. The Atakr Multicenter Investigators Group. Circulation 1999; 99 :262-70

2. Cappato R, Calkins H, Chen SA, et al. Worldwide survey on the methods, efficacy, and safety of catheter ablation for human atrial fibrillation. Circulation 2005;111:1100-5.

3. Segal OR, Chow AW, Markides V, et al. Long-term results after ablation of infarct-related ventricular tachycardia. Heart Rhythm 2005;2:474-82.

4. Nahass GT. Fluoroscopy and the skin: implications for radiofrequency catheter ablation. Am J Cardiol 1995:76:174-6.

5. Park TH, Eichling JO, Schechtman KB, et al. Risk of radiation induced skin injuries from arrhythmia ablation procedures. Pacing Clin Electrophysiol 1996;19:1363-9.

6. Lickfett L, Mahesh M, Vasamreddy C, et al. Radiation exposure during catheter ablation of atrial fibrillation. Circulation 2004;110:3003-10.

7. Kovoor $\mathbf{P}$, Ricciardello $\mathrm{M}$, Collins $\mathrm{L}$, et al. Risk to patients from radiation associated with radiofrequency ablation for supraventricular tachycardia. Circulation 1998;98:1534-40.

8. Perisinakis K, Damilakis J, Theocharopoulos N, et al. Accurate assessment of patient effective radiation dose and associated detriment risk from radiofrequency catheter ablation procedures. Circulation 2001;104:58-62.

9. Damilakis J, Theocharopoulos N, Perisinakis K, et al. Conceptus radiation dose and risk from cardiac catheter ablation procedures. Circulation 2001;104:893-7.

10. Cheema A, Dong J, Dalal D, et al. Long-term safety and efficacy of circumferential ablation with pulmonary vein isolation. J Cardiovasc Electrophysiol 2006;17:1080-5.

11. Theocharopoulos $\mathbf{N}$, Damilakis J, Perisinakis K, et al. Occupational exposure in the electrophysiology laboratory: quantifying and minimizing radiation burden. $\mathrm{Br} J \mathrm{Radio}$ 2006;79:644-51.

12. Lloyd $\mathbf{P}$, Lowe D, Harty DS, et al. The secondary radiation grid; its effect on fluoroscopic dose-area product during barium enema examinations. $\mathrm{Br} \mathrm{J}$ Radiol 1998; 71:303-6.

13. Tapiovaara M. PCXMC: A PC-based Monte Carlo program for calculating patient doses in medical x-ray examinations. Report No: STUK-A139. Helsinki: Finnish Centre for Radiation and Nuclear Safety. 1997. 9 A.D.

14. Servomaa A, Tapiovaara M. Organ dose calculation in medical $x$ ray examinations by the program PCXMC. Radiation Prot Dosimetry 1998;80:213-19.

15. Draft recommendations of the international commission on radiological protection. 2007 http://www.icrp.org/docs/ICRP_Draft_Recommendations_12_January_2007. pdf (accessed 14 Jun 2007).

16. Le Heron JC. Estimation of effective dose to the patient during medical x-ray examinations from measurements of the dose-area product. Phys Med Biol 1992;37:2117-26.

17. Theocharopoulos N, Perisinakis K, Damilakis J, et al. Comparison of four methods for assessing patient effective dose from radiological examinations. Med Phys 2002:29:2070-9

18. McFadden SL, Mooney RB, Shepherd PH. X-ray dose and associated risks from radiofrequency catheter ablation procedures. Br J Radiol 2002;75:253-65.

19. Efstathopoulos EP, Katritsis DG, Kottou S, et al. Patient and staff radiation dosimetry during cardiac electrophysiology studies and catheter ablation procedures: a comprehensive analysis. Europace 2006:8:443-8.

20. Davies AG, Cowen AR, Kengyelics SM, et al. X-ray dose reduction in fluoroscopically guided electrophysiology procedures. Pacing Clin Electrophysiol 2006;29:262-71.

21. Onnasch DG, Schemm A, Kramer HH. Optimization of radiographic parameters for paediatric cardiac angiography. Br J Radiol 2004;77:479-87.

22. Partridge J, McGahan G, Causton $\mathrm{S}$, et al. Radiation dose reduction without compromise of image quality in cardiac angiography and intervention with the use of a flat panel detector without an antiscatter grid. Heart 2006; 92:507-10.

23. Rotter M, Takahashi $Y$, Sanders $P$, et al. Reduction of fluoroscopy exposure and procedure duration during ablation of atrial fibrillation using a novel anatomica navigation system. Eur Heart J 2005;26:1415-21.

24. Calkins H, Niklason L, Sousa J, et al. Radiation exposure during radiofrequency catheter ablation of accessory atrioventricular connections. Circulation 1991;84:2376-82.

25. Wittkampf FH, Wever EF, Vos K, et al. Reduction of radiation exposure in the cardiac electrophysiology laboratory. Pacing Clin Electrophysiol 2000;23:1638-44.

26. Macle L, Weerasooriya $\mathrm{R}$, Jais $\mathrm{P}$, et al. Radiation exposure during radiofrequency catheter ablation for atrial fibrillation. Pacing Clin Electrophysiol 2003;26:288-91.

27. Food and Drug Administration. Important Information for Physicians and Other Healthcare Professionals: Recording Information in the Patient's Medical Record That Identifies the Potential for Serious X-ray-Induced Skin Injuries Following Fluoroscopically Guided Procedures. Rockville, MD, Center for Devices and Radiological Health. FDA, 1995.

28. Hughes JS, Watson SJ, Jones AL, et al. Review of the radiation exposure of the UK population. J Radiol Prot 2005;25:493-6.

29. den BA, de Feyter PJ, Hummel WA, et al. Reduction of radiation exposure while maintaining high-quality fluoroscopic images during interventional cardiology using novel x-ray tube technology with extra beam filtering. Circulation 1994;89:2710-14

30. Rosenthal LS, Mahesh M, Beck TJ, et al. Predictors of fluoroscopy time and estimated radiation exposure during radiofrequency catheter ablation procedures. Am J Cardiol 1998;82:451-8. 